\title{
Asymptotic modelling of a six-hole MOF
}

\author{
Michael J. Chen, Yvonne M. Stokes, Peter Buchak, Darren G. Crowdy, and Heike Ebendorff-Heidepriem
}

\begin{abstract}
We model the drawing of a six-hole microstructured optical fibre with a combination of asymptotic techniques and a new efficient numerical method, and compare this to a previous set of experiments and finite element simulations. The new approach accurately models the deformation of the inner channels and predicts cross-sectional fibre geometries which are a better match to the experiments than the previous, more computationally expensive simulation technique.
\end{abstract}

Index Terms-Microstructured optical fibres (MOFs); fibre drawing; mathematical modelling; asymptotic modelling.

\section{INTRODUCTION}

$\mathbf{M}$ ICROSTRUCTURED optical fibres (MOFs) are an emerging technology which have already found novel uses in a wide range of fields, for instance in biological sensing, supercontinuum generation and high speed communications [1]. They are tailored to suit a specific application by the design of the cross-sectional pattern of the internal air channels which run along their length, thus altering the refractive index of the fibre from that of pure glass. Their fabrication involves heating and drawing a large scale preform to fibre dimensions (see Fig. 1), and a major challenge here is that the relative size and shape of the internal channels may deform dramatically during this process. Typically trial and error experiments are run to determine the combination of preform design and operational parameters (furnace temperature, draw speed, channel pressurisation) required to achieve a desired fibre design; this can be a lengthy and expensive process. For example, it might be desirous to choose parameters to give a fibre which replicates the preform design at a smaller scale or to apply a large channel pressurisation to produce a fibre with air channels that are separated by very thin struts of glass. Modelling of the fabrication is therefore of great practical interest, since this provides predictive tools to understand how the channels deform over a range of conditions.

The study of Luzi et al. [2] is a compelling demonstration of how a model can predict deformation in MOF drawing. Here a six-hole preform was drawn to fibre over a range of channel pressurisations and then compared to finite element

This research was supported by grant DP130101541 from the Australian Research Council and by a Research Grant from the Leverhulme Trust in the United Kingdom.

Michael Chen is with the Mathematical Institute, University of Oxford, Oxford, OX2 6GG, UK (email: chenm@maths.ox.ac.uk).

Yvonne Stokes is with the School of Mathematical Sciences, The University of Adelaide, Adelaide, SA 5005, Australia (email yvonne.stokes@adelaide.edu.au)

Peter Buchak and Darren Crowdy are with the Department of Mathematics, Imperial College London, London, SW7 2AZ, UK (email: p.buchak@imperial.ac.uk; d.crowdy@imperial.ac.uk).

Heike Ebendorff-Heidepriem is with the ARC Centre of Excellence for Nanoscale BioPhotonics, Institute for Photonics and Advanced Sensing, School of Physical Sciences, The University of Adelaide, Adelaide, SA 5005, Australia (email: heike.ebendorff@adelaide.edu.au).

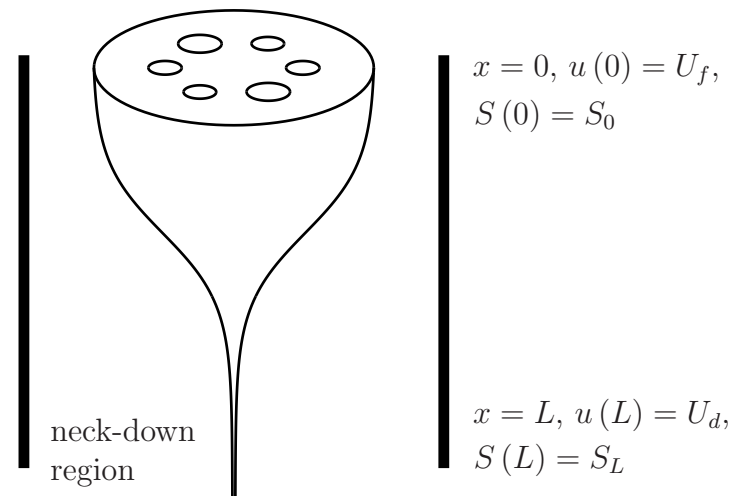

Fig. 1. Schematic of the fibre drawing process where a preform is heated and stretched to the dimensions of a fibre.

simulations performed with the commercial package ANSYS Polyflow. The results showed promising agreement when the cross-sectional shape of the channels remained circular, although at larger pressurisations, where significant deformation had occured, the simulation overestimated the cross-sectional area of each channel.

The computations in [2] were performed with a finiteelement package; whilst [2] does not give details about how long each computation took to run, it is typical that even the most efficient finite element code of this type will require a run-time (at least) in the order of hours. The disadvantage to such intensive simulations is that they are costly to rerun with slightly different parameters. For instance, in [2] it is possible that even better results may have been obtained with a different temperature profile. Indeed, although such simulations are appropriate for comparative studies, the usefulness of modelling this process is much greater where the operational parameters can be changed easily.

The modelling approach of the present study has been developed by the authors [3], [4] (building on earlier work in [5]-[7], among others) specifically for the problem of fibre drawing. This approach immediately has an advantage over a package which is, by nature, designed to be as general as possible in scope and not context specific. Our approach uses perturbation techniques which exploit the slenderness of the neck-down shape and a suite of efficient, newly developed numerical techniques [8], [9]. These give detailed understanding of how the internal structures of MOFs deform during drawing under the competing influences of surface tension, viscous stresses in the glass as it is stretched (or drawn) under tension from preform to fibre dimensions, and channel pressurisation. A key point of difference between our asymptotic approach and previous asymptotic approaches is that it can treat a MOF of any internal geometry, that is to 
say, the limitations of asymptotic models noted in [2], that they could not treat eccentrically located channels or the interaction between neighbouring channels, have been overcome.

The model has already been validated against experiments where tubular preforms were drawn to fibre under a range of conditions [10] and was generally an excellent match for these simple geometries. Other studies have similarly demonstrated the utility of asymptotic models for drawing tubular fibres [11], [12] and for multi-holed geometries with channels separated by thin struts [13]. The motivation for the present study is to compare the model to an existing experiment in the literature which has a more complicated cross-sectional structure than a simple tube. This is a precursor to future, more thorough validation against new purpose-designed experiments.

We provide more detail on the modelling approach in section 2. The relevant details of the experiment of Luzi et al. [2] are given in section 3, where we demonstrate the calculation of viscosity and surface tension parameters which are important to the modelling approach. The asymptotic model is compared to both the experimental measurements and finite element simulations from [2] in section 4.

\section{ASYMPTOTIC MODELLING APPROACH}

A schematic of the fibre drawing process is shown in Fig. 1. Here a preform with some cross-sectional pattern of channels (typically of a few centimetres in diameter and around 10 to $20 \mathrm{~cm}$ in length) is lowered into a furnace where it is heated, drawn to fibre dimensions (around 100 to $200 \mu \mathrm{m}$ in diameter) and then wound round a drum. The preform is fed into the furnace at speed $U_{f}$ and the drum rotates at a much faster draw speed $U_{d}$. The ratio between these two speeds is the draw ratio $D$ and by mass conservation it must also be the case that

$$
U_{d} / U_{f}=S_{0} / S_{L}=D
$$

where $S_{0}$ is the cross-sectional area of the preform and $S_{L}$ is the cross-sectional area of the fibre. The other crucial operational parameters are the fibre tension $\mathcal{T}$, by convention given as a weight in grams, and internal pressurisation $p_{H}$. The important material properties of the glass are its surface tension $\gamma$ and its viscosity profile through the neck-down $\mu(x)$.

For the purposes of the modelling we introduce a dimensionless fibre tension parameter $\mathcal{T}^{*}$, a dimensionless pressurisation parameter $\mathcal{P}^{*}$ and a dimensionless surface tension parameter $\gamma^{*}$. These are defined in terms of the dimensional quantities as

$$
\begin{aligned}
\mathcal{T}^{*} & =\frac{\mathcal{T} \times 9.81 \times 10^{-3}}{6 \gamma \sqrt{S_{0}}}, \quad \mathcal{P}^{*}=\frac{p_{H} \sqrt{S_{0}}}{\gamma}, \\
\gamma^{*} & =\frac{\gamma L}{\mu_{0} U_{f} \sqrt{S_{0}}},
\end{aligned}
$$

where $L$ is the length of the neck-down region and $\mu_{0}$ is a viscosity scale such that $\mu(x)=\mu_{0} \mu^{*}(x)$, and here $\mu^{*}$ is a dimensionless version of the viscosity profile. As in [3], it is
TABLE I

SUMMARY OF VARIOUS QUANTITIES AND THEIR UNITS

quantity
$U_{f}$
$U_{d}$
$S_{0}$
$S_{L}$
$D$
$\mathcal{T}$
$p_{H}$
$\gamma$
$\mu(x)$
$L$
$\mu_{0}$
$\mu^{*}(x)$
$\mathcal{T}^{*}$
$\mathcal{P}^{*}$
$\gamma^{*}$
$x$
$x^{*}$
$\tau$
$\chi$
$\Gamma$
$\mathbf{U}=v \hat{\mathbf{j}}+w \mathbf{k}$
$p$
$\hat{\mathbf{n}}$
$\sigma$
$G$
$T(x)$

$$
\begin{gathered}
\text { description } \\
\text { feed speed } \\
\text { draw speed } \\
\text { preform cross-sectional area } \\
\text { fibre area cross-sectional area } \\
\text { draw ratio } \\
\text { fibre tension } \\
\text { channel pressurisation } \\
\text { surface tension } \\
\text { neck-down viscosity profile } \\
\text { neck-down length } \\
\text { viscosity scale } \\
\text { dimensionless viscosity profile } \\
\text { dimensionless tension parameter } \\
\text { dimensionless pressurisation parameter } \\
\text { dimensionless surface tension parameter } \\
\text { physical axial co-ordinate }(0 \leq x \leq L) \\
\text { dimensionless axial co-ordinate }\left(0 \leq x^{*} \leq 1\right) \\
\text { Lagrangian reduced time co-ordinate } \\
\text { square-root of cross-sectional area } \\
\text { total circumference of all boundaries } \\
\text { cross-plane velocity vector } \\
\text { cross-plane pressure } \\
\text { cross-plane normal vector } \\
\text { Cauchy stress tensor }(2 \mathrm{D}) \\
\text { free surface } \\
\text { neck-down temperature profile }
\end{gathered}
$$

convenient to choose $\mu_{0}$ as the inverse harmonic mean of the viscosity profile over the neck-down, namely

$$
\mu_{0}=L / \int_{0}^{L} \frac{1}{\mu(x)} \mathrm{d} x
$$

The various parameters and dimensionless quantities are summarised in table I.

Our approach assumes the shape of the neck-down is slender, that is $\epsilon=\sqrt{S_{0}} / L \ll 1$, and this significantly simplifies the full three-dimensional Navier-Stokes equations. As outlined in detail in [3] and [4], we make various perturbation expansions in $\epsilon^{2}$ and solve a coupled system of equations for the leading-order flow in the axial direction (that is, along the axis of the fibre) and for the flow in the cross-plane (in every cross-section along the length of the neck-down).

We introduce a Lagrangian reduced-time co-ordinate $\tau$ as the independent variable. This measures the time since the start of deformation of a cross-section (accounting for the various scalings and the viscosity profile) and is, therefore, also a measure of the distance travelled along the neck-down length from $x=0$ to $x=L$. In this formulation the conservation of axial momentum is written in an integrated form

$$
\frac{\mathrm{d} \chi}{\mathrm{d} \tau}=\frac{\chi}{12} \Gamma(\tau)-\mathcal{T}^{*},
$$

where $\Gamma(\tau)$ is the total circumference of all boundaries in the cross-plane (see below) and $\chi(\tau)$ is the square-root of crosssectional area along the neck-down, both these quantities being scaled by the square-root of the preform area $\sqrt{S_{0}}$. To convert from the Lagrangian co-ordinate back to physical space it 


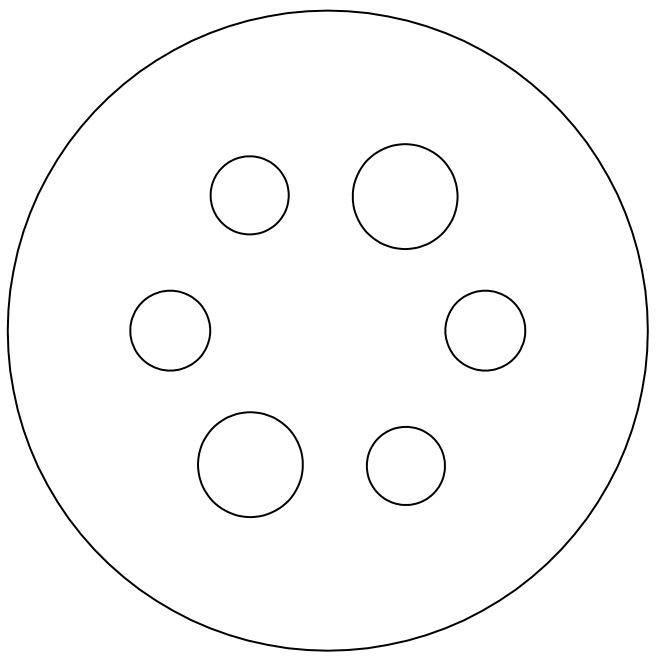

Fig. 2. Schematic of the preform geometry. The preform outer diameter is $3.93 \mathrm{~mm}$. The six internal holes are each described by their diameter $D$ and their position $(x, y)$, where the centre of the outer boundary is $(x, y)=(0,0)$. Clockwise from the large top right hole, the six holes are $(D, x, y)=(611,484,822),(469,968,1.12),(458,477,-828)$, $(611,477,823),(468,-966,-0.986)$ and $(459,-477,827) \mu \mathrm{m}$.

is necessary to solve an equation for the axial co-ordinate, namely

$$
\frac{\mathrm{d} x^{*}}{\mathrm{~d} \tau}=\frac{\mu^{*}\left(x^{*}\right)}{\gamma^{*} \chi},
$$

where we have introduced a dimensionless axial co-ordinate $x^{*}=x / L$. In the cross-plane we solve a $2 \mathrm{D}$ Stokes-flow free boundary problem. Due to the scaling this is solved in a domain of unit area, with unit surface tension and viscosity. The conservation of mass and momentum in the fluid are

$$
\begin{aligned}
\nabla_{\perp} \cdot \mathbf{U} & =0, \\
-\nabla_{\perp} p+\nabla_{\perp}^{2} \mathbf{U} & =0,
\end{aligned}
$$

where $\mathbf{U}=v \hat{\mathbf{j}}+w \hat{\mathbf{k}}$ (here $v$ and $w$ are the velocities in the $y$ and $z$ directions, respectively), $p$ is the pressure in the fluid region and $\nabla_{\perp}=\partial / \partial y \mathbf{j}+\partial / \partial z \mathbf{k}$. The stress and kinematic boundary conditions on the free surface $G(x, y, z)=0$ are

$$
\begin{aligned}
\sigma \cdot \hat{\mathbf{n}} & =-\left(\kappa+\mathcal{P}^{*} \chi\right) \hat{\mathbf{n}}, \\
\frac{\mathrm{d} G}{\mathrm{~d} \tau}+v \frac{\mathrm{d} G}{\mathrm{~d} y}+w \frac{\mathrm{d} G}{\mathrm{~d} z} & =0,
\end{aligned}
$$

where $\sigma$ is the Cauchy stress tensor, $\kappa$ is the curvature of the free surface and the unit normal vector is $\hat{\mathbf{n}}=\nabla_{\perp} G /\left|\nabla_{\perp} G\right|$. The axial and cross-plane models are coupled via the $\Gamma$ term in (5), which must be obtained from the solution to the crossplane model (7)-(10), and via the $\mathcal{P}^{*} \chi$ term in (9), where $\chi$ is obtained from the solution to the axial model.

A variety of methods can be used to solve the cross-plane problem depending on the geometry. There is an analytic solution for an unpressurised tube [3], for example, and we have previously made use of an efficient computational scheme where the cross-sectional shape of the channels are ellipses [8]. For the simulations in this paper we use a spectral method [9] that can handle very general channel shapes and compute their evolution accurately and efficiently. In this method, the shape of the cross section is described by a time-varying conformal map from a multiply connected circular domain to the cross section, with a circular inner boundary mapping to the boundary of each channel. Initially, the map is trivial because the channels in the preform are all circular. The method calculates the evolution of both the map and the locations and sizes of the domain's boundaries to determine the evolution of the cross section. Equations (7)-(8) are satisfied by introducing a pair of analytic functions that determine a biharmonic stream function. At each time step, (9) determines the flow field that results in the correct stresses on the boundary, while (10) is used to calculate the time derivatives of the map and domain in order to update the shape of the cross section.

In our previous experimental study [10] we measured fibre tension directly, but did not impose a specific viscosity profile in the model. This meant the model output consisted of the final geometry of the fibre and the dimensionless parameter $\gamma^{*}$, from which the harmonic mean of viscosity could be inferred via (3). In the simulations in [2], there is a prescribed viscosity profile but no information on the fibre tension which requires a slightly different approach to the model. In this situation it is necessary to iteratively solve for the dimensionless tension $\mathcal{T}^{*}$ with a fixed value of $\gamma^{*}$ determined by the viscosity profile. In practice this involves solving the model from $x^{*}=0$ to $x^{*}=1$, iteratively adjusting the value of $\mathcal{T}^{*}$ until the crosssectional area of the fibre (at $x^{*}=1$ ) is $\chi^{2}=1 / D$, as required by mass conservation.

\section{LUZI ET AL.'S SIX-HOLE EXPERIMENT}

A schematic of the preform geometry to be used in the simulations is shown in Fig. 2; here we have used MATLAB's Image Processing Toolbox to obtain the dimensions from the picture of the meshed preform geometry given in [2] so that our initial condition will be consistent with that used in the finite element simulations of that work. The preform has an outer diameter of $3.93 \mathrm{~mm}$ and features six internal holes, the exact dimensions and positions of which are given in the caption to Fig. 2. The cross-sectional glass area of the preform is $S_{0}=1.088 \times 10^{-5} \mathrm{~m}^{2}$.

The feed speed for the experiment was $U_{f}=20 \mathrm{~mm} / \mathrm{min}$ and the draw speed was $U_{d}=18.7 \mathrm{~m} / \mathrm{min}$, for a draw ratio of $D=935$. The preform is made of synthetic silica glass which has a surface tension of $\gamma=0.3 \mathrm{~N} / \mathrm{m}$. Pressurisation was varied from $p_{H}=0$ to $300 \mathrm{mbar}$ in increments of $50 \mathrm{mbar}$.

The furnace temperature used was $1890^{\circ} \mathrm{C}$. Several assumptions were made for the purposes of the finite element simulations, namely that the length of the hot-zone over which the deformation takes place is $L=0.1 \mathrm{~m}$ and that the temperature profile over this zone is

$$
T(x)=a \exp (x-b)^{2} /\left(2 c^{2}\right),
$$

with $a=1890^{\circ} \mathrm{C}, b=L / 2=0.05 \mathrm{~m}$ and $c=0.03$ for $x<b$, or $c=0.028$ for $x \geq b$. This Gaussian profile is plotted against $x$ over the length of the hot-zone in Fig. 3, and note here that the shape of this curve is slightly different to the equivalent plot given in [2], where that plot was for different values of $c$ which did not match the stated values in the text. 


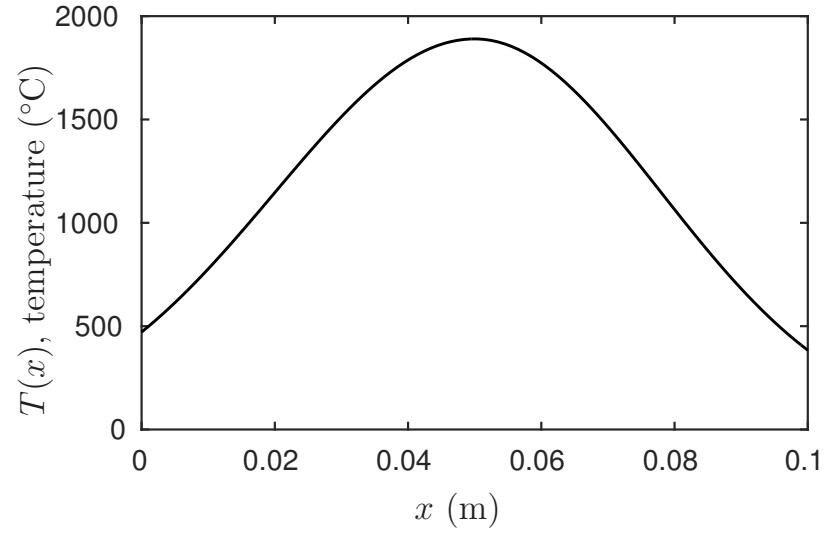

Fig. 3. Temperature profile of the neck-down region, as specified by (11). This is an asymmetric Gaussian profile with a peak temperature of $1890^{\circ} \mathrm{C}$. The other parameters are given in the text below (11).

The relationship between temperature and viscosity for silica glass is given in [14] as

$$
\mu(T)=5.8 \times 10^{-8} \times \exp \left(\frac{515400}{8.3145 T+2271.10567}\right) \mathrm{Pa} \mathrm{s}
$$

and so we can calculate the viscosity profile $\mu(x)$ by substituting (11) into (12). From (4), the harmonic mean of viscosity is

$$
\mu_{0}=L / \int_{0}^{L} \frac{1}{\mu(x)} \mathrm{d} x=1.13 \times 10^{6} \mathrm{~Pa} \mathrm{~s},
$$

which is then used to calculate the the dimensionless surface tension parameter $\gamma^{*}=2.43 \times 10^{-2}$ from (3).

\section{RESUlTS AND DISCUSSION}

Our numerical scheme involves solving a system of differential equations made up of (5)-(6) along with equations for the evolution of each free surface. These latter equations are given in [9] and we refer interested readers to that work for full details of this efficient spectral method. For the simulations presented here 20 spectral coefficients were used to represent each free-surface, which is more than sufficient to capture the expected deformations. The whole system was then solved with the Runge-Kutta $(4,5)$ method ODE45 in MATLAB 8.5 (R2015a).

Where the fibre tension is known, a single run of the simulation takes around 50 seconds on a laptop with modest specifications (Intel Core i7-2620M Processor and $8 \mathrm{~GB}$ of RAM). Since fibre tension was not measured in [2] we iteratively solved for this quantity with a bisection method routine until the ratio between the measured preform and computed fibre area $S_{0} / S_{L}$ converged to within $10^{-6}$ of the required draw ratio $D$. This required at most 20 iterations of the bisection method for each value of pressurisation, with a typical total run time of about 15 minutes.

Four cross-sectional fibre geometries from the asymptotic simulation are compared with the results from [2] in Fig. 4. Here we have reproduced Fig. 3 from [2], where the finite element simulation is overlaid in light transparent blue. The results of the asymptotic solution are shown as thin red lines; note that the outer boundaries of the fibre cross-sections in all the photographs are not visible since they are hidden behind the overlaid asymptotic results.

A key aspect of these results to note is that, as calculated from the assumed temperature/viscosity profile (11)-(12), this experiment was performed at relatively high tension, as compared to the experiments reported in [10], for instance. The pressures applied to the holes are also orders of magnitude larger than in that work. As discussed in detail in [2]-[4], the high viscous stresses in the glass associated with such large values of fibre tension will dominate any effects due to surface tension or small pressurisation. This dominance is reflected in the very large values of pressure which were required in these experiments to achieve significant inflation and deformation of the internal channels. The calculated tension values increase slightly as the pressure is increased; at $p_{H}=0$ the tension is $85.65 \mathrm{~g}$ and for $p_{H}=300 \mathrm{mbar}$ the tension is $85.68 \mathrm{~g}$.

The agreement between the experiments and the asymptotic model is qualitatively excellent, as shown by the close match between the photographs of the four fibre cross-sections and the overlaid solutions in Fig. 4. For the unpressurised case shown in Fig. 4(a), the photograph, finite element simulation and the asymptotic results are nearly indistinguishable, in fact the relative size and shape of the holes are nearly unaltered from the those of the preform. Here the fibre tension is so dominant that there is no discernable deformation due to surface tension.

For the remaining examples in Figs. 4(b)-(d), where successively larger hole pressurisations are applied, the match between the photographs and the asymptotic solution is excellent, with the simulation broadly agreeing with the size and rounded triangular shape of the internal holes. By contrast, the finite element simulation tends to overestimate the size of the internal holes as more pressure is applied. This suggests that the method used by the finite element scheme is less precise in capturing the interplay between surface tension and pressurisation.

For larger pressurisations the internal holes are no longer circular. Their ultimate shape is due to a combination of the applied pressurisation, surface tension and the influence of the neighbouring channels. As they evolve through the neck-down away from being perfectly circular the curvature of each channel boundary $\kappa$ is no longer uniform around each boundary. Thus the effect of surface tension in (9) is highly localised and to accurately capture this the curvature of the free surfaces must be calculated with a high degree of precision. The spectral method from [9] calculates this quantity exactly, while inaccuracies may be introduced in a finite element method through the approximation of higherorder derivatives.

The outer diameter measurements of the fibre for all seven values of pressurisation used in the experiments and corresponding values for both the finite element (taken from [2, Fig. 4]) and asymptotic simulations are shown in Fig. 5. As with the results shown in Fig. 4, there is excellent agreement between the measured outer diameter and the asymptotic 

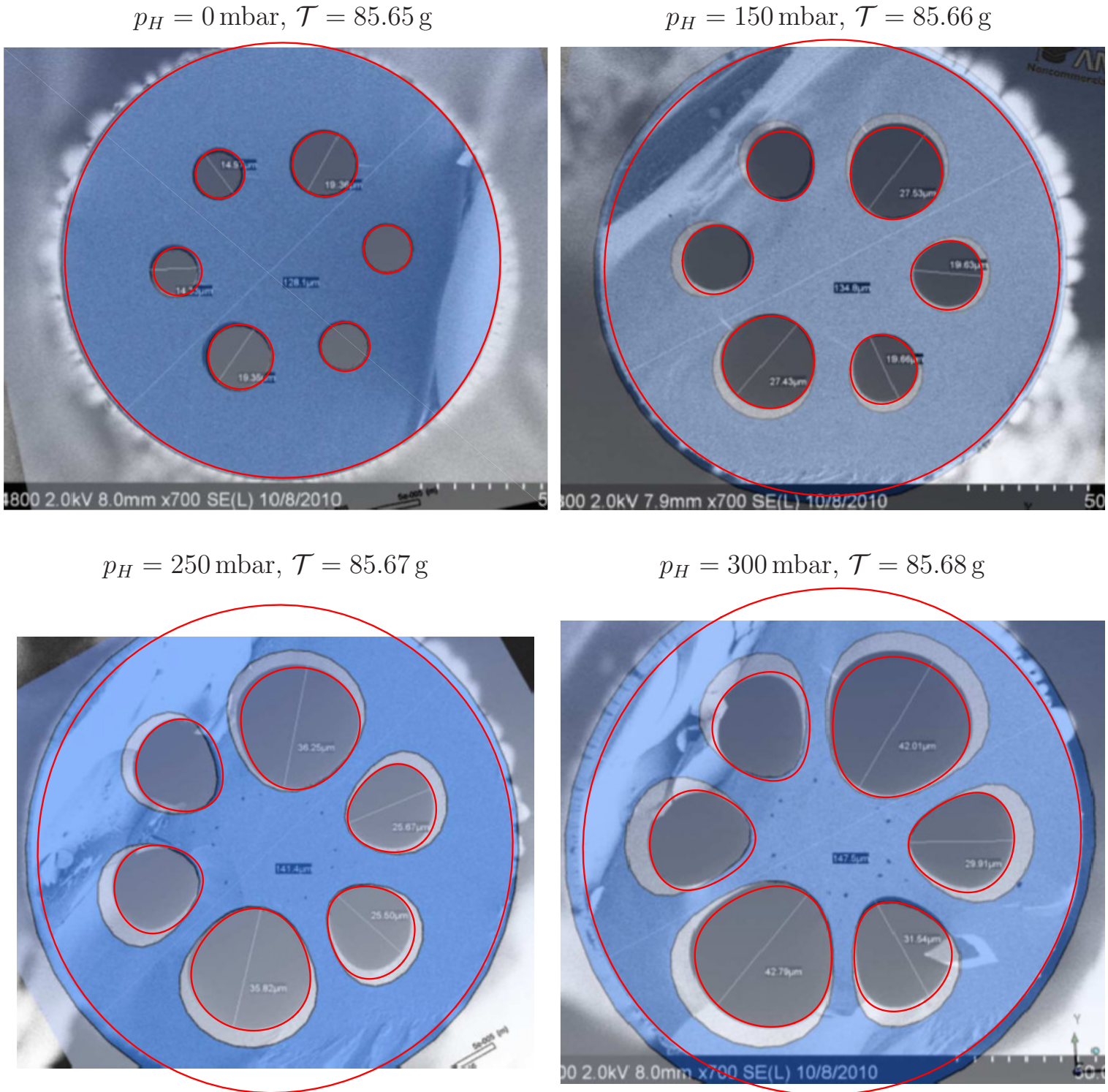

Fig. 4. Experimental microscope images of the fibre cross-section, overlaid with the finite element simulation of [2] (pale blue transparency) and the results of the new asymptotic simulation (thin red lines). Shown are the four values of pressurisation from [2, Fig. 3]. For each example the pressurisation applied is shown in the caption above the image, along with the fibre tension as calculated by the iterative scheme.

simulation. Here, the particularly close match at large pressurisation, where the internal holes have undergone significant expansion and deformation, is an improvement over the finite element simulation. Note that measurement error for this type of microscopy is typically in the order of a few microns and the finite element simulations are within this error for the first three or four measurements.

A similar plot for the maximum extent (longest distance between any two points on a particular boundary) of the large and small holes is shown in Fig. 6, along with the data for the experimental measurements and finite element simulations from [2, Fig. 5]. Each point here is the mean value of the two large or the four small holes, respectively, at each pressurisation value. Note that the four small holes are initially two pairs of holes of slightly different diameters, but are averaged together here to allow a comparison with [2].
Again, there is excellent agreement at all pressures between the experimental values and the asymptotic model. The discrepancies here between the experiments and finite element simulations are revealing. For instance, the initial curvature $\kappa$ of the larger holes is less than that of the smaller holes, thus at identical pressurisation the Young-Laplace balance in (9) will be weighted more in favour of pressurisation for larger holes. The differences between the observed values and the finite element simulations are significant even at moderate pressurisations for the larger holes, which suggests that curvature is underestimated by the finite element approach.

\section{CONCLUSION}

The asymptotic model of microstructured optical fibre drawing [3], [4], [9] predicts cross-sectional fibre geometries that 


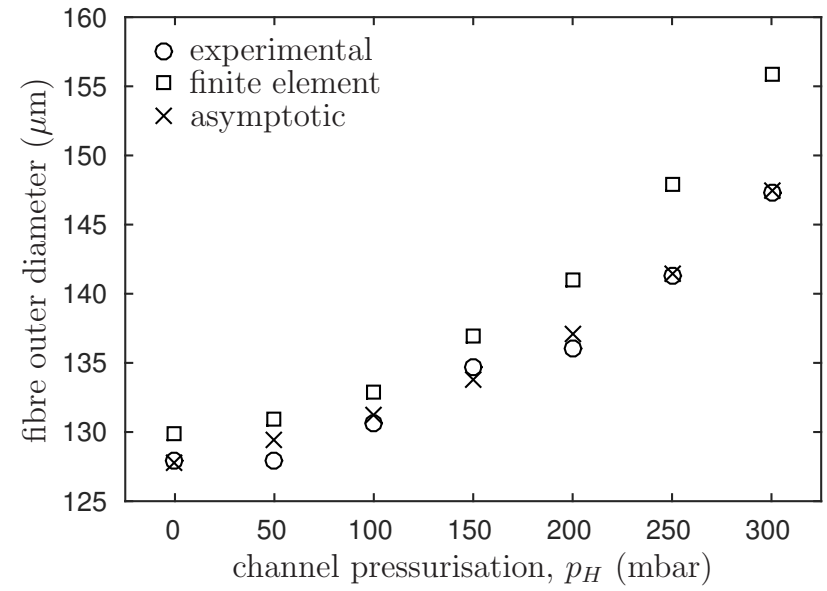

Fig. 5. Fibre outer diameter for the seven values of pressurisation used in the experiment, where the experimental measurements and finite element simulation from [2, Fig. 4] are compared with the asymptotic model.

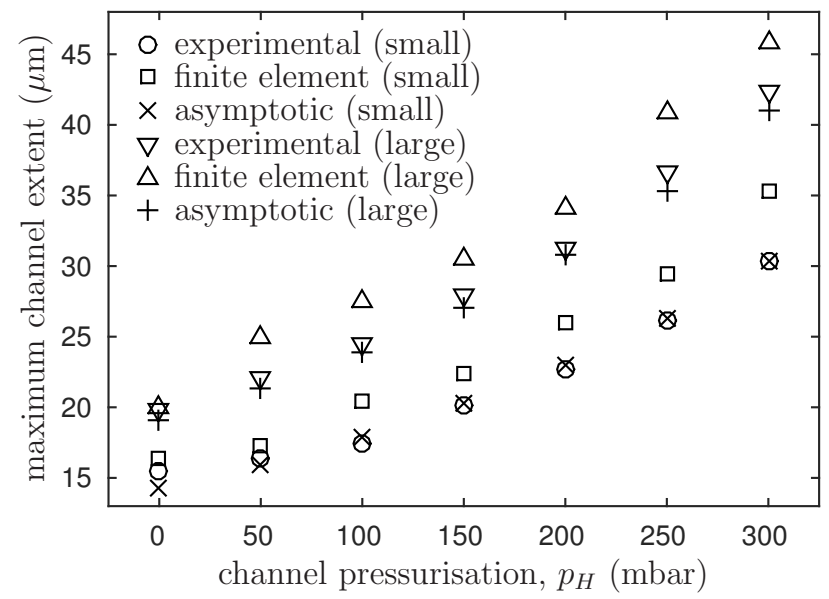

Fig. 6. The maximum extent of the internal holes versus the seven values of pressurisation, where the experimental measurements and finite element simulations from [2, Fig. 5] are compared with the asymptotic model. Results for the large and small holes are shown separately and have been averaged so that the 'large' points are the mean value of the two large holes and 'small' points are the mean value of the four small holes.

are an excellent match with the experimental results in [2]. Indeed, this new simulation approach is significantly closer to the experiments, and is more computationally efficient, than the finite element simulations in [2], where both approaches use identical initial conditions and temperature/viscosity profiles. Specifically, the internal holes in the asymptotic simulation are not as inflated as in the finite element simulation, because the interfacial curvature (and thus the effect of surface tension) is calculated exactly by the spectral method from [9].

This success of the asymptotic method is surprising since our original intention for this study was to match our technique to the finite element simulations, rather than the experimental results, with the goal of showing that our more computationally efficient method could replicate the output of a commercially available package. Our results show that precise calculation of the interfacial deformation is crucial to the predictive abilities of fibre-drawing models, and suggest that full three-dimensional modelling of the glass temperature, as proposed in [2], is not necessary.

A key assumption made by both modelling approaches is the form of the axial temperature profile. As demonstrated by [3], making any such assumption about the unknown temperature profile can be avoided by measuring fibre tension during a draw. Further modelling of this profile is crucial if it is to be fully understood, since it is clear from the experiments on tubular drawing in [10] that it depends on both peak furnace temperature and the geometry of the internal channels over the neck-down region. Future efforts will concentrate on modelling this axially varying temperature; it may be possible to develop a model for drawing MOFs by extending previous studies of simpler geometries, for instance the asymptotic approaches to the temperature modelling of solid fibre drawing [15] or the stretching of heated tubes [16]. Future purpose designed experiments will focus on validating the asymptotic model for multi-hole preforms with larger outer diameters than the one used in Luzi et al. [2]; this will test the limits of the modelling assumption that the neck-down region is slender. We will also consider preforms with asymmetry in their crosssectional structure to directly investigate whether cross-plane temperature variation is present.

\section{REFERENCES}

[1] T. M. Monro and H. Ebendorff-Heidepriem, "Progress in microstructured optical fibers," Ann. Rev. Materials Res., vol. 36, pp. 467-495, 2006.

[2] G. Luzi, P. Epple, M. Scharrer, K. Fujimoto, C. Rauh, and A. Delgado, "Numerical Solution and Experimental Validation of the Drawing Process of Six-Hole Optical Fibers Including the Effects of Inner Pressure and Surface Tension," J. Lightwave Technol., vol. 30, no. 9, pp. 13061311, 2012.

[3] Y. M. Stokes, P. Buchak, D. G. Crowdy, and H. Ebendorff-Heidepriem, "Drawing of micro-structured fibres: circular and non-circular tubes," $J$. Fluid Mech., vol. 755, pp. 176-203, 2014.

[4] M. J. Chen, Y. M. Stokes, P. Buchak, D. G. Crowdy, and H. EbendorffHeidepriem, "Microstructured optical fibre drawing with active channel pressurisation," J. Fluid Mech., vol. 783, pp. 137-165, 2015.

[5] L. Cummings and P. Howell, "On the evolution of non-axisymmetric viscous fibres with surface tension, inertia and gravity," J. Fluid Mech., vol. 389, pp. 361-389, 1999.

[6] I. M. Griffiths and P. D. Howell, "The surface-tension-driven evolution of a two-dimensional annular viscous tube," J. Fluid Mech., vol. 593, pp. 181-208, 2007.

[7] - "Mathematical modelling of non-axisymmetric capillary tube drawing," J. Fluid Mech., vol. 605, pp. 181-206, 2008.

[8] P. Buchak, D. G. Crowdy, Y. M. Stokes, and H. Ebendorff-Heidepriem, "Elliptical pore regularization of the inverse problem for microstructure optical fibre fabrication," J. Fluid Mech., vol. 778, pp. 5-38, 2015.

[9] P. Buchak and D. G. Crowdy, "Surface-tension-driven Stokes flow: A numerical method based on conformal geometry," J. Comput. Phys., vol. 317, pp. 347-361, 2016.

[10] M. J. Chen, Y. M. Stokes, P. Buchak, D. G. Crowdy, H. T. C. Foo, A. Dowler, and H. Ebendorff-Heidepriem, "Drawing tubular fibres: experiments versus mathematical modelling," Opt. Mater. Express, vol. 6, no. 1, pp. 166-180, 2016.

[11] A. D. Fitt, K. Furusawa, T. M. Monro, C. P. Please, and D. J. Richardson, "The mathematical modelling of capillary drawing for holey fibre manufacture," J. Eng. Math., vol. 43, pp. 201-227, 2002.

[12] G. Luzi, P. Epple, M. Scharrer, K. Fujimoto, C. Rauh, and A. Delgado, "Comparison Between an Analytical Asymptotic Fiber Drawing Model With Full Navier-Stokes Solution Taking Into Account the Effects of Inner Pressure and Surface Tension," J. Lightwave Technol., vol. 29, no. 11 , pp. 1638-1646, 2011.

[13] G. T. Jasion, J. S. Shrimpton, Y. Chen, T. Bradley, D. J. Richardson, and F. Poletti, "MicroStructure Element Method (MSEM): viscous flow model for the virtual draw of microstructured optical fibers," Opt. Express, vol. 23, no. 1, pp. 312-329, 2015. 
[14] G. Urbain, Y. Bottinga, and P. Richet, "Viscosity of liquid silica, silicates and alumino-silicates," Geochimica Cosmochimica Acta, vol. 46, no. 6, pp. 1061-1072, 1982.

[15] M. Taroni, C. Breward, L. Cummings, and I. M. Griffiths, "Asymptotic solutions of glass temperature profiles during steady optical fibre drawing," J. Eng. Math., vol. 80, pp. 1-20, 2013.

[16] H. Huang, J. J. Wylie, R. M. Miura, and P. D. Howell, "On the formation of glass microelectrodes," SIAM J. Appl. Math., vol. 67, no. 3, pp. 630666, 2007. 